\title{
Predictors of Successful First-Pass Thrombectomy with a Balloon Guide Catheter: Results of a Decision Tree Analysis
}

\author{
Aglaé Velasco Gonzalez $^{1}$ (D) Dennis Görlich ${ }^{2} \cdot$ Boris Buerke $^{1} \cdot$ Nico Münnich $^{1} \cdot$ Cristina Sauerland $^{2} \cdot$ Thilo Rusche $^{1}$. \\ Andreas Faldum $^{2} \cdot$ Walter Heindel ${ }^{1}$
}

Received: 9 October 2019 / Revised: 13 January 2020 / Accepted: 28 January 2020 / Published online: 23 May 2020

(C) The Author(s) 2020

\begin{abstract}
Complete recanalization after a single retrieval maneuver is an interventional goal in acute ischemic stroke and an independent factor for good clinical outcome. Anatomical biomarkers for predicting clot removal difficulties have not been comprehensively analyzed and await unused. We retrospectively evaluated 200 consecutive patients who suffered acute stroke and occlusion of the anterior circulation and were treated with mechanical thrombectomy through a balloon guide catheter (BGC). The primary objective was to evaluate the influence of carotid tortuosity and BGC positioning on the one-pass Modified Thrombolysis in Cerebral Infarction Scale (mTICI) 3 rate, and secondarily, the influence of communicating arteries on the angiographic results. After the first-pass mTICI 3, recanalization fell from 51 to $13 \%$. The regression models and decision tree (supervised machine learning) results concurred: carotid tortuosity was the main constraint on efficacy, reducing the likelihood of mTICI 3 after one pass to $30 \%$. BGC positioning was relevant only in carotid arteries without elongation: BGCs located in the distal internal carotid artery (ICA) had a $70 \%$ probability of complete recanalization after one pass, dropping to $43 \%$ if located in the proximal ICA. These findings demonstrate that first-pass mTICI 3 is influenced by anatomical and interventional factors capable of being anticipated, enabling the BGC technique to be adapted to patient's anatomy to enhance effectivity.
\end{abstract}

Keywords Stroke $\cdot$ Thrombectomy $\cdot$ Suction $\cdot$ Carotid arteries $\cdot$ Circle of Willis

Abbreviations
ACA Anterior cerebral artery
ACoA Anterior communicating artery
BGC Balloon guide catheter
Electronic supplementary material The online version of this article
(https://doi.org/10.1007/s12975-020-00784-2) contains supplementary
material, which is available to authorized users.

Aglaé Velasco Gonzalez

Aglae.VelascoGonzalez@ukmuenster.de

Dennis Görlich

Dennis.Goerlich@ukmuenster.de

Boris Buerke

buerkeb@uni-muenster.de

Nico Münnich

Nico.Muennich@klinikumdo.de; nico.muennich@web.de

Cristina Sauerland

sauerla@uni-muenster.de

Thilo Rusche

Thilo.Rusche@ukmuenster.de

\author{
CCA Common carotid artery \\ CI Confidence interval \\ CoW Circle of Willis \\ CTA Computed tomography angiography
}

\author{
Andreas Faldum \\ Andreas.Faldum@ukmuenster.de \\ Walter Heindel \\ heindel@uni-muenster.de
}

1 Department of Clinical Radiology, Institute of Clinical Radiology and Neuroradiology, University Hospital of Muenster, Albert-Schweitzer-Campus 1, Building A1, 48149 Muenster, Germany

2 Institute of Biostatistics and Clinical Research, University of Muenster, Schmeddingstraße 56, 48149 Muenster, Germany 


$\begin{array}{ll}\text { DSA } & \text { Digital subtraction angiography } \\ \text { ICA } & \text { Internal carotid artery } \\ \text { MT } & \text { Mechanical thrombectomy } \\ \text { mTICI } & \text { Modified Thrombolysis in Cerebral Infarction Scale } \\ \text { NIHSS } & \text { National Institutes of Health Stroke Scale } \\ \text { OR } & \text { Odds ratio } \\ \text { PCA } & \text { Posterior cerebral artery } \\ \text { PCoA } & \text { Posterior communicating artery } \\ \text { PH } & \text { Parenchymal hematoma } \\ \text { SR } & \text { Stent retriever } \\ \text { tPA } & \text { Tissue plasminogen activator }\end{array}$

\section{Introduction}

In acute ischemic stroke, clinical outcomes depend heavily on rapid and complete recanalization [1-4]. The goal for interventional therapies is thus complete recanalization after a single retrieval maneuver, which is an independent factor for good clinical outcome [5]. Combining stent retrievers (SRs) and balloon guide catheters (BGCs) is a commonly used endovascular clot removal technique [6]. This mechanical thrombectomy (MT) technique requires antegrade flow to be arrested (temporal occlusion of the carotid artery) and simultaneous aspiration through the BGC for retrieval (flow reversal) [7-9]. Unfortunately, the chances of achieving Modified Thrombolysis in Cerebral Infarction Scale (mTICI) 3 decrease with each retrieval maneuver $[5,10,11]$. Consequently, it is essential to analyze the anatomical and angiographic factors that could alter the effectiveness of clot removal on the first attempt using the combined SR-BGC technique. These biomarkers indicative of the difficulty of clot removal could be identified prior to therapy, thereby enabling the mechanical thrombectomy technique to be adapted to each patient's anatomical conditions.

Few studies have evaluated the effect of angiographic factors such as carotid tortuosity on recanalization rates, with marked disparity in their results. Yilmaz et al. [12] used computed tomography angiography (CTA) to study the influence of carotid elongation on recanalization (mTICI $\geq 2 \mathrm{~b}$ independent of the number of passes) in a series of 54 MTs but failed to show any impact of elongation on the angiographic results. In contrast, more recently, Jeong et al. [13] examined the frequency of successful recanalization ( $\mathrm{mTICI} \geq 2 \mathrm{~b}$ ) in the presence of carotid tortuosity and BGC placement for therapy (distally versus proximally in the internal carotid artery (ICA)), but they did not provide any related statistical test results. Although the rate of successful recanalization was higher in the group with the combination of "tortuosity absent" and distal BGC positioning in the ICA, the published data do not provide statistical confirmation that carotid elongation significantly influences BGC positioning or significantly affects the rate of successful recanalization [13]. To the best of our knowledge, the effect of carotid tortuosity on the position of the BGC for treatment and on recanalization rates has not yet been determined. Thus, greater insight into the relationships among all these factors is required, focusing on the best angiographic outcome attainable, complete recanalization (mTICI 3) after one SR pass.

A further matter to consider is whether the presence of communicating arteries could influence MT outcomes. In normal circumstances, the middle cerebral artery (MCA) and the anterior cerebral artery (ACA) are supplied mainly by the ICA. However, in cases of acute occlusion, flow direction and volume through the communicating arteries may change in response to the new cerebral perfusion requirements [14-16]. Reversal of communicating artery flow to the occlusion side results in a supplementary continuous blood supply that should also be aspirated through the BGC. Thus, the suction effect from aspiration through the BGC under conditions of flow arrest in the ICA is enhanced when there are no communicating arteries and could hypothetically decrease when these arteries are present [7].

The objective of this analysis was to assess the influence of carotid elongation, BGC positioning, and anatomical variations of the circle of Willis (CoW) on complete recanalization rates after one pass in 200 consecutive patients who underwent mechanical thrombectomy with BGC. Additionally, the National Institutes of Health Stroke Scale (NIHSS) scores were correlated with the angiographic findings.

\section{Methods}

\section{General}

The primary objective of this study was to assess the association between carotid elongation and BGC location for therapy and its impact on one-pass complete recanalization. Secondarily, the presence of communicating arteries ipsilateral to the stroke side and their influence on the angiographic results and clot migration during therapy were also analyzed. This retrospective study was approved by our institution's review board. Informed consent was waived. This study received no industry support.

\section{Patient Selection}

Between January 2016 and December 2018, 283 patients were treated consecutively for acute ischemic stroke of the anterior circulation by MT with SR through a BGC as the first-choice technique, in all cases using flow arrest and continuous manual aspiration during the retrieval maneuver. Patients from this group with tandem stenosis/occlusions $(n=47)$ or clot extension into the extracranial carotid artery $(n=36)$ were 
excluded, resulting in a total study group of 200 patients for this retrospective analysis.

\section{Assessment of the Results}

We retrospectively compiled and analyzed the clinical patient data, including stroke risk factors, stroke demographics, and prior treatment with intravenous tissue plasminogen activator (tPA). NIHSS scores at presentation and at discharge were obtained from Neurology Department records. One NIHSS score at presentation and six scores at discharge went unrecorded. In addition, 33 patients died while in hospital; their final NIHSS scores were not documented.

The anatomy of the CoW ipsilateral to the stroke side was determined from the invasive angiographic images on record and correlated with the features of the CTA images to obtain a final evaluation. Variations in diameters between the MCA and the ICA were determined as the ratio of the diameter of the ICA (measured distal to the posterior communicating artery (PCoA) segment) to that of the proximal M1 segment. Elongation of the carotid artery was defined on the basis of such angiographic features as the presence of kinking or tortuosity of the ICA distal to the position of the BGC (Fig. 1), as proposed by Jeong et al. [13]. BGC positioning for treatment was classified into three groups based on dividing the ICA into three segments: (1) the distal ICA (BGC tip located in the distal third of the ICA, i.e., subpetrosal placement), (2) the proximal ICA (BGC tip located in the caudal two thirds of the ICA), and (3) the distal common carotid artery (CCA) (Fig. 2).

All patients were treated using an SR + microcatheter through an 8-French BGC (flow arrest + manual aspiration during retrieval) as the first-choice technique. Selection of the SR device was left to the operator. The procedure time, defined as the time elapsing between the first angiographic image obtained using a guide catheter in the extracranial carotid artery and the end of SR recanalization, was also recorded [8]. The degree of postintervention vessel recanalization was based on the mTICI score, whereby grade $2 b$ indicates at least $50 \%$ reperfusion of the affected region and grade 3 complete reperfusion. For the purpose of this analysis, cases of mTICI grade $2 \mathrm{c}$ were included in the mTICI 3 population. Complete recanalization after a single retrieval maneuver (first-pass effect) [5] was defined as achieving mTICI 3 after a single pass with the SR. Successful revascularization was defined as a final mTICI grade of at least $2 b$ on conclusion of the procedure after up to three passes. Postinterventional mTICI grades were assigned by the treating physician at the end of the intervention in the clinical setting. The grades were re-evaluated retrospectively by a second neuroradiologist (AVG) to obtain a final assessment; discrepancies were settled by consensus. Any suspicion of clot migration during therapy necessitated retrospective evaluation of the CTA to rule out multiple emboli in the distal territories at presentation.
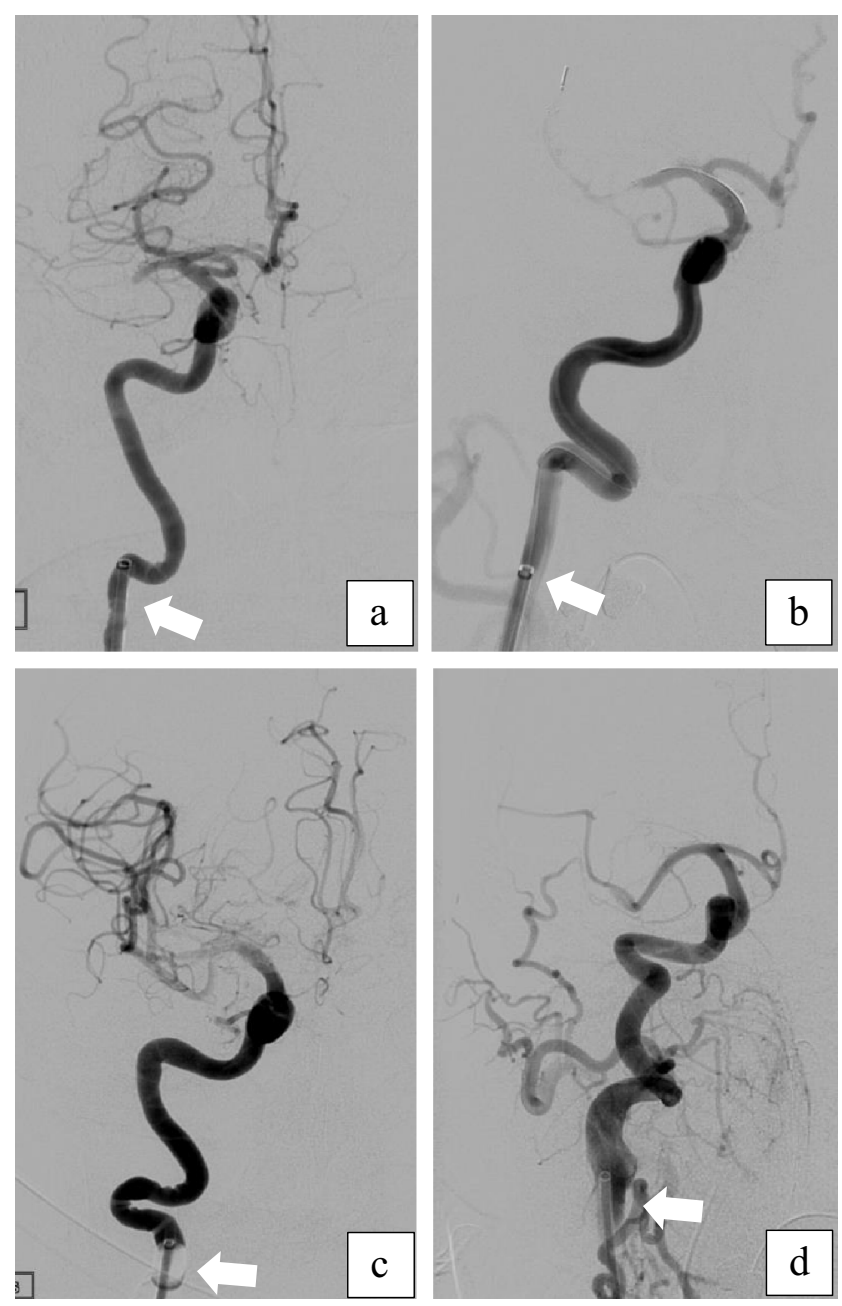

Fig. 1 a-d Frontal and oblique views of carotid elongation using digital subtraction angiography (DSA). Four examples of internal carotid artery (ICA) elongation distal to the tip of the balloon guide catheter (BGC). In all these cases, the BGC was placed in the proximal ICA (dividing the ICA into three segments from the subpetrosal segment to the extracranial bifurcation, the proximal ICA is the caudal two thirds of the cervical carotid). Case A shows occlusion of the middle cerebral artery and a single $90^{\circ}$ kink. Case B shows double ICA kinking and an open retriever device in the distal M1 segment into the M2 segment through a distal M1-M2 clot. Cases C and D depict multiple ICA kinks (the follow-up angiography after recanalization in case $\mathrm{C}$ was inadvertently performed under balloon inflation)

Follow-up imaging (CT) was performed after $24 \mathrm{~h}$. Adverse procedure-related events were recorded. Hemorrhage was graded according to the method used in the European Cooperative Acute Stroke Trials [17].

\section{Statistical Analysis}

Three of the authors were involved in the statistical analysis (AVG (neuroradiologist), DG (statistician), and CS (statistician)). The Mann-Whitney $U$ test, chi-square test, and Fisher's exact test were used to compare the continuous and categorical baseline variables between the first-pass mTICI 3 
Fig. 2 Balloon guide catheter position (BGC) in the carotid artery. From right to left, angiographic classification of BGC location for treatment: dividing the internal carotid artery (ICA) into three segments from the subpetrosal segment to the extracranial bifurcation, the proximal ICA is the caudal two thirds of the cervical carotid. The following two images show distal BGC positioning (a) and proximal BGC positioning (b) in the ICA

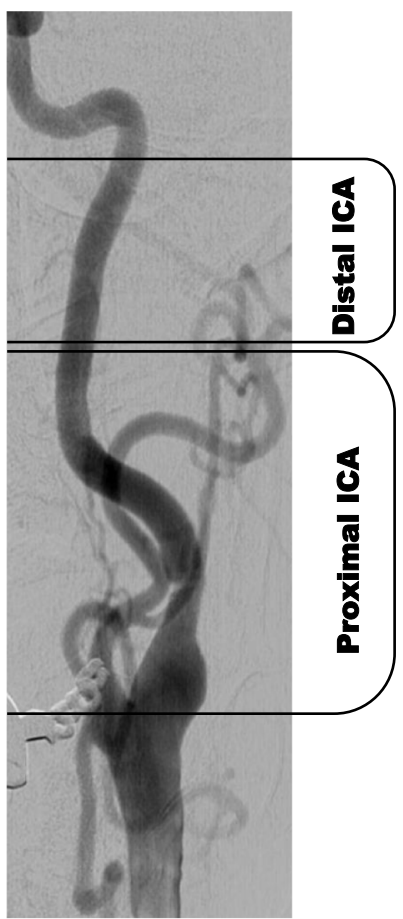

groups (yes/others). Changes in the initial and final NIHSS scores were compared using the Wilcoxon signed-rank test.

The association between carotid elongation (yes/no) and BGC position was evaluated using the chi-square test. Additionally, the association between elongation and BCG position with first-pass mTICI 3 and successful recanalization was analyzed using univariate and multivariable logistic regression models while adjusting for stroke laterality, intravenous thrombolysis, previous anticoagulation, ICA/MCA ratio, intervention duration, and ipsilateral complete CoW. Wald test $p$ values were recorded together with the odds ratio (OR) ( $95 \%$ confidence intervals (CIs)). $p$ values $\leq 0.05$ were deemed to indicate a statistically significant difference. All reported $p$ values were two-sided. Finally, a decision tree was implemented to predict one-pass complete recanalization based on all the variables, according to the following settings: maximum depth of 4 , minimum splitting size of 10 , minimum child size of 5, 10-fold cross-validation, alpha of 0.05 , and splitmerged. This analysis was Bonferroni adjusted. SPSS (version 25; IBM, Armonk, NY, USA) was used for all statistical analyses.

\section{Results}

\section{Demographics}

This study included 115 women (mean age, $78 \pm 12$ years; age range, 31-96) and 85 men (mean age, $70 \pm 15$ years, age range, 32-96) $(p<0.0001)$. The baseline characteristics of

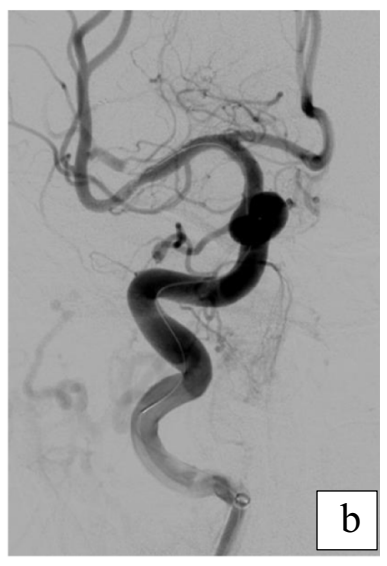

$\mathrm{b}$

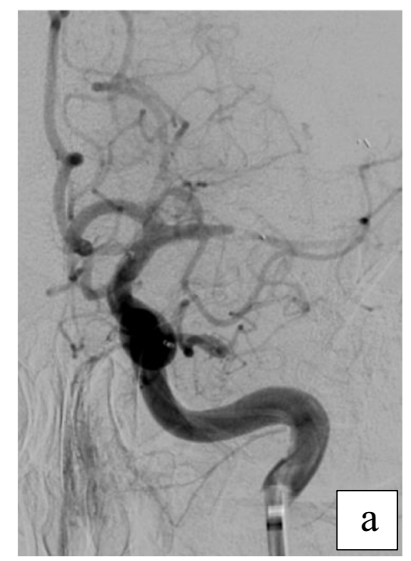

the 200 patients in whom complete recanalization (mTICI 3) was achieved with a single retrieval maneuver are presented in Table 1. The median initial NIHSS score was 15 (interquartile range (IQR) 11-17), decreasing significantly at discharge (median final NIHSS score (IQR) $3(2-7) ; p<0.0001$ ).

Several types of SR were used, most frequently the preset 4 $(n=144)$ (Preset SR; Phenox, Bochum, Germany) and the preset $6(n=37)$. In most cases, a single SR was used for thrombectomy $(85 \%, 170 / 200)$. Multiple SRs (15\%, 30/200) were associated with significantly longer intervention times (median duration of MT with multiple SRs, IQR $60 \mathrm{~min}$ (40-80) versus $17 \mathrm{~min}(14-26)$ with a single SR; $p<0.0001$ ) (see the supplemental material, Table 1: types of SRs used).

In the non-enhanced follow-up CT scan after $24 \mathrm{~h}$, hemorrhagic complications with mass effect (parenchymal hematoma type 2 (PH-2)) were observed in six cases (3\%), hemorrhagic complications without mass effect (parenchymal hematoma type 1 (PH-1)) in 21 cases (10.5\%), and subarachnoid hemorrhage in 20 cases $(10 \%)$. The inpatient mortality rate was $21 \%(41 / 200)$. The logistic regression showed that inhospital mortality in the elderly was higher than that in younger patients, with the risk increasing by a factor of 1.034 (1.004-1.066) per year $(p=0.028)$.

\section{Per-Pass Achievement of $\mathbf{m T I C I} 3$}

Recanalization was successful (mTICI $3 / 2 \mathrm{~b}$ after up to three passes) in $83.5 \%$ of cases $(167 / 200)$. Irrespective of the number of passes, mTICI 3 was achieved in $62.5 \%$ of cases (125/ 
Table 1 Patient demographics, occlusions, and interventions

\begin{tabular}{|c|c|c|c|c|}
\hline & \multirow[t]{2}{*}{ Total $(n=200)$} & \multicolumn{2}{|c|}{ Complete one-pass recanalization } & \multirow[t]{2}{*}{$p$ value } \\
\hline & & Yes $(n=102)$ & No $(n=98)$ & \\
\hline Gender, female, $n(\%)$ & 115 & $57(55.9)$ & $58(59.2)$ & 0.0162 \\
\hline Age, years (IQR) & $77(70,84)$ & $76(68,81)$ & $78(71,85)$ & 0.678 \\
\hline Hypertension, $n(\%)$ & $120(60)$ & $55(53.9)$ & $65(66.3)$ & 0.073 \\
\hline Diabetes mellitus, $n(\%)$ & $35(17.5)$ & $21(20.6)$ & $14(13.7)$ & 0.241 \\
\hline Smoker, $n(\%)$ & $24(12)$ & $14(13.7)$ & $10(10.2)$ & 0.444 \\
\hline \multicolumn{5}{|l|}{ Atrial fibrillation, $n(\%)$} \\
\hline $\begin{array}{l}\text { New diagnosis } \\
\text { Previous diagnosis }\end{array}$ & $\begin{array}{l}49(24.5) \\
50(25)\end{array}$ & $\begin{array}{l}28(27.5) \\
26(25.5)\end{array}$ & $\begin{array}{l}21(21.4) \\
24(24.5)\end{array}$ & 0.536 \\
\hline Previous anticoagulation therapy, $n(\%)$ & $85(42.5)$ & $46(45.1)$ & $39(39.8)$ & 0.048 \\
\hline Dyslipidemia, $n(\%)$ & $42(21)$ & $21(21.4)$ & $21(21.4)$ & 0.884 \\
\hline \multicolumn{5}{|l|}{ Stroke demographics } \\
\hline \multicolumn{5}{|l|}{ Stroke laterality, $n(\%)$} \\
\hline $\begin{array}{l}\text { Right } \\
\text { Left }\end{array}$ & $\begin{array}{l}102(51) \\
98(49)\end{array}$ & $\begin{array}{l}60(58.8) \\
42(42.9)\end{array}$ & $\begin{array}{l}42(41.2) \\
56(57.1)\end{array}$ & 0.024 \\
\hline IV thrombolysis & $109(54.5)$ & $55(53.9)$ & $54(55.1)$ & 0.867 \\
\hline \multicolumn{5}{|l|}{ Location of the occlusion, $n(\%)$} \\
\hline $\begin{array}{l}\text { ICA } \\
\text { Proximal } \mathrm{M}^{\dagger}\end{array}$ & $\begin{array}{l}31(15.5) \\
67(33.5)\end{array}$ & $\begin{array}{l}17(16.7) \\
38(37.3)\end{array}$ & $\begin{array}{l}14(14.3) \\
29(29.6)\end{array}$ & \multirow[t]{4}{*}{0.201} \\
\hline Distal M1 ${ }^{\dagger}$ & $65(32.5)$ & $32(31.4)$ & $33(33.7)$ & \\
\hline Proximal M2 & $29(14.5)$ & $14(13.7)$ & $15(15.3)$ & \\
\hline Distal M2 & $8(4)$ & $7(7.1)$ & $1(1)$ & \\
\hline $\begin{array}{l}\text { Initial median NIHSS score (IQR) } \\
\text { NA }\end{array}$ & $\begin{array}{l}15(11-17) \\
1\end{array}$ & $\begin{array}{l}15(11-17) \\
1\end{array}$ & $\begin{array}{l}15(11-17) \\
-\end{array}$ & 0.966 \\
\hline $\begin{array}{l}\text { Final median NIHSS score }{ }^{\$} \text { (IQR) } \\
\text { NA }^{\dagger}\end{array}$ & $\begin{array}{l}3(2-7) \\
39\end{array}$ & $\begin{array}{l}3(2-7) \\
15\end{array}$ & $\begin{array}{l}4(2-7) \\
24\end{array}$ & 0.379 \\
\hline \multicolumn{5}{|l|}{ Final NIHSS score - initial NIHSS score } \\
\hline $\begin{array}{l}\text { Median (IQR) } \\
\text { NA }\end{array}$ & $\begin{array}{l}9(5-13) \\
40\end{array}$ & $\begin{array}{l}9(5-13) \\
16\end{array}$ & $\begin{array}{l}10(5-12) \\
24\end{array}$ & 0.795 \\
\hline In-hospital mortality, $n(\%)$ & $42(21)$ & $17(16.7)$ & $25(25.5)$ & 0.164 \\
\hline
\end{tabular}

In the statistical test, the chi-square test was used for categorical variables and the Mann-Whitney test for ordinal variables. Data have been expressed as median values (interquartile range (IQR)) or numbers (no.) (percentage (\%))

ICA internal carotid artery, NIHSS National Institutes of Health Stroke Scale

${ }^{\dagger} \mathrm{M} 1$ segment of the middle cerebral artery (MCA)

${ }^{\star} \mathrm{M} 2$ segment of the MCA

${ }^{\$}$ Final NIHSS score was not available (NA) for 39 patients, including 33 deaths during hospitalization, and was unrecorded in 6 cases. Units in parentheses are percentages or ranges; error is expressed as $\pm \mathrm{SD}$

200). In 102 of 200 cases (51\%), complete recanalization was achieved with one pass. Figure 3 summarizes the angiographic mTICI 3 rates achieved per pass. The chances of mTICI 3 were reduced to one fifth after the first pass.

\section{Elongation and BGC Positioning}

Positioning of the BGC for therapy depended on the presence or absence of carotid elongation $(p<0.0001)$. The absence of carotid elongation increased the likelihood of the BGC being placed more distally in the ICA and vice versa. Rates of carotid elongation with respect to the position of the BGC for therapy are set out in Table 2.

According to the univariate analysis, one-pass complete recanalization depended significantly on the presence of carotid elongation and the $\mathrm{BGC}$ position (see Table 3 ). However, according to the multivariate model, carotid elongation was the only significant factor associated with mTICI 3 after a single pass. A straight carotid artery (no elongation) increased the likelihood of complete recanalization in one pass by a factor of 2.410 (95\% CI, 1.138-5.104; $p=0.0216)$. Table 4 outlines the effect of BGC positioning, carotid elongation, 
Fig. 3 Changes in mTICI 3 rates per stent retriever pass

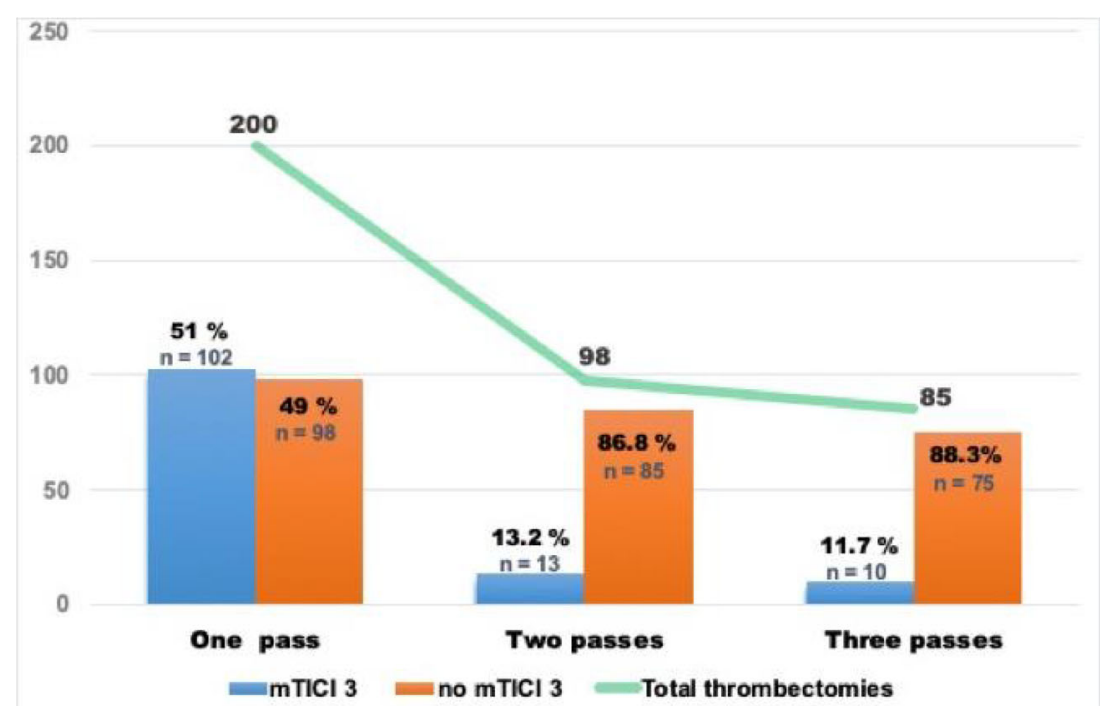

mTICI: modified Thrombolysis in Cerebral Infarction Scale lateralization and location of the occlusion, and intravenous thrombolysis on complete recanalization after one pass.

To estimate the effect of BGC position, we performed a subgroup analysis on the thrombectomies without carotid elongation $(n=117)$. Distal placement of the BGC in the ICA was an independent factor for complete recanalization after a single SR pass (reference: proximal ICA location; OR 3.188 ; 95\% CI, $1.163-8.740 ; p=0.024)$, as was the laterality of the occlusion (reference: left side; OR 2.619; 95\% CI, $1.151-5.956 ; p=0.022$ ).

The best exploratory conditions for achieving one-pass mTICI 3 based on the interaction term were as follows: BGC in the distal ICA + no elongation ( $68 \%$ probability), followed by $\mathrm{BGC}$ in the proximal ICA + no elongation (45\%); BGC in the proximal ICA + elongation (45\%); BGC in the distal ICA + elongation (33\%); and BGC in the CCA + elongation $(16 \%)$.

\section{Decision Tree Analysis}

To verify the findings, a machine learning decision tree was developed to ascertain the relevant variables for predicting complete recanalization after the first pass. In addition, the decision tree could potentially enable further refinement of patient classification, as appropriate, by adding other variables, always maintaining a minimum significance level of 0.05 between subgroups of patients. As depicted in Fig. 4, the decision tree confirms and summarizes the abovementioned results of the regression analysis.

Carotid tortuosity was selected as the first variable for predicting complete recanalization after one pass. In the presence of carotid elongation, the likelihood of one-pass complete recanalization was $32.5 \%(p<0.0001)$. If the carotid artery was straight, the position of the BGC in the ICA was the most important factor for predicting the outcome. If the BGC was positioned in the distal ICA, the probability of complete recanalization from one pass was almost $70 \%$ as opposed to $43 \%$ if placed in the proximal ICA $(p=0.021)$.

\section{Circle of Willis Anatomy}

Figure 1 in the supplemental material presents the frequencies of anatomical variations in the $\mathrm{CoW}$. The presence of an anterior communicating artery $(\mathrm{ACoA})$ was the most common

Table 2 Carotid artery tortuosity and BGC positioning for mechanical thrombectomy

\begin{tabular}{llll}
\hline Balloon guide catheter Location & Total $(n=200), n(\%)$ & Carotid artery tortuosity & $p$ value \\
\cline { 3 - 4 } & & Yes $(n=83), n(\%)$ & No $(n=117), n(\%)$ \\
\hline Distal ICA & $109(54.5)$ & $15(18.1)$ & $94(80.3)$ \\
Proximal ICA & $82(41)$ & $59(71.1)$ & $23(19.7)$ \\
Distal common carotid artery & $9(4.5)$ & $9(10.8)$ & 0 \\
\hline
\end{tabular}

Two-sided Fisher's exact test

ICA internal carotid artery 
Table 3 Univariate analysis of anatomical and interventional characteristics in cases of complete one-pass recanalization

\begin{tabular}{|c|c|c|c|c|}
\hline & \multicolumn{3}{|c|}{ Complete one-pass recanalization } & \multirow[t]{2}{*}{$p$ value } \\
\hline & Total $(n=200)$ & Yes $(n=102)$ & No $(n=98)$ & \\
\hline \multicolumn{5}{|l|}{ Retrieval conditions } \\
\hline Ipsilateral ACoA and PCoA, $n(\%)$ & $45(22.5)$ & $18(40)$ & $27(60)$ & 0.170 \\
\hline \multicolumn{5}{|l|}{ ICA/MCA ratio } \\
\hline $\begin{array}{l}\text { Median (IQR) } \\
\text { NA }\end{array}$ & $\begin{array}{l}1.4(1.3,1.5) \\
12\end{array}$ & $\begin{array}{l}1.4(1.3,1.5) \\
8\end{array}$ & $\begin{array}{l}1.4(1.3,1.56) \\
4\end{array}$ & 0.678 \\
\hline Elongation, $n(\%)$ & $83(41.5)$ & $27(26.5)$ & $56(57.1)$ & $<0.0001$ \\
\hline \multicolumn{5}{|l|}{ Intervention characteristics } \\
\hline Duration of intervention (min), median value (IQR) & $19(15-36)$ & $16(14-18)$ & $35(22-59)$ & $<0.0005$ \\
\hline \multicolumn{5}{|l|}{ BGC position, $n(\%)$} \\
\hline $\begin{array}{l}\text { Distal ICA } \\
\text { Proximal ICA }\end{array}$ & $\begin{array}{l}109(54.5) \\
82(41)\end{array}$ & $\begin{array}{l}70(68.6) \\
31(30.4)\end{array}$ & $\begin{array}{l}39(39.8) \\
51(52)\end{array}$ & \multirow[t]{2}{*}{$<0.0001$} \\
\hline Distal common carotid artery & $9(4.5)$ & $1(1)$ & $8(8.2)$ & \\
\hline Migration to new territory, $n(\%)$ & $6(3)$ & $2(2)$ & $4(4.1)$ & 0.379 \\
\hline
\end{tabular}

In the statistical test, the chi-square test was used for categorical variables and the Mann-Whitney test for ordinal variables. Data have been expressed as median values (interquartile range (IQR) or numbers $(n)$ (percentage $(\%)$ )

$A C O A$ anterior communicating artery, $P C O A$ posterior communicating artery, $I C A$ internal carotid artery, $M C A$ middle cerebral artery

anatomical variant $(76.5 \%, 153 / 200)$. Coexistence of an ACoA and a PCoA was observed in $22.5 \%$ of cases (45/ 200). The rate of mTICI 3 on the first pass did not differ significantly in cases of a coexisting ACoA and PCoA ipsilateral to the stroke side $(60 \%, 27 / 45)$ as compared with all other anatomical variations combined $(48.4 \%, 75 / 155)(p=0.170)$.

Table 4 Multiple logistic regression for predicting complete one-pass recanalization in mechanical thrombectomies through a balloon guide catheter (BGC)

\begin{tabular}{lll}
\hline & OR $(95 \% \mathrm{CI})$ & $p$ value \\
\hline BGC position & & \\
Distal ICA & $1.903(0.913-3.967)$ & 0.086 \\
Common carotid artery & $0.352(0.040-3.076)$ & 0.352 \\
$\quad$ Proximal ICA (ref) & - & \\
Carotid elongation & & 0.022 \\
$\quad$ Yes & - & \\
No (ref) & $1.860(1.011-3.423)$ & 0.046 \\
Lateralization of occlusion & - & \\
Right & & \\
Left (ref) & $1.103(0.479-2.543)$ & 0.817 \\
Location of occlusion & - & \\
ICA & & \\
MCA (ref) & $1.039(0.564-1.915)$ & 0.902 \\
IV thrombolysis & - & \\
Yes &
\end{tabular}

ICA internal carotid artery, $M C A$ middle cerebral artery, $C I$ confidence interval
Migration into a new territory occurred in six cases (five into the ACA and one into the posterior cerebral artery (PCA)). Four of these cases were treated with low-profile $\mathrm{SR}$ (for $\leq 3$-mm vessels). In the logistic regression adjusted by CoW anatomy, the ICA/MCA ratio, intravenous thrombolysis, and intervention duration, the presence of an ACoA was an independent factor that afforded protection against migration into a new territory (reference: absent ACoA or contralateral A1 segment; OR 0.051; 95\% CI, 0.004-0.667; $p=0.023$ ). Furthermore, the risk of migration significantly increased by a factor of 3.579 (OR) with every $30 \mathrm{~min}$ of intervention time (95\% CI, 1.558-8.224; $p=0.0027$ ).

\section{Discussion}

Single-pass mTICI 3 rates achieved by MT using an SR through a $\mathrm{BGC}$ ranged from $30 \%$ to a maximum of $70 \%$ depending on anatomical and interventional factors. Carotid elongation was the most potent biomarker for predicting an ineffective first pass $(67 \%$ likelihood of non-mTICI 3 outcome). The more proximal or distal positioning of the BGC in the ICA initially appeared to be very promising, based on the univariate analysis results, but when adjusted to account for other factors, this factor no longer proved to be significant. Machine learning (decision tree) helped in interpreting the tangled relationship between carotid tortuosity and BGC positioning. Beyond the obvious impediments to advancing the BGC distally in cases of carotid tortuosity, we were able to identify a more decisive role of BGC location for therapy in cases of a straight carotid artery (no tortuosity). In this setting, 
First pass $\mathrm{mTICl} 3$

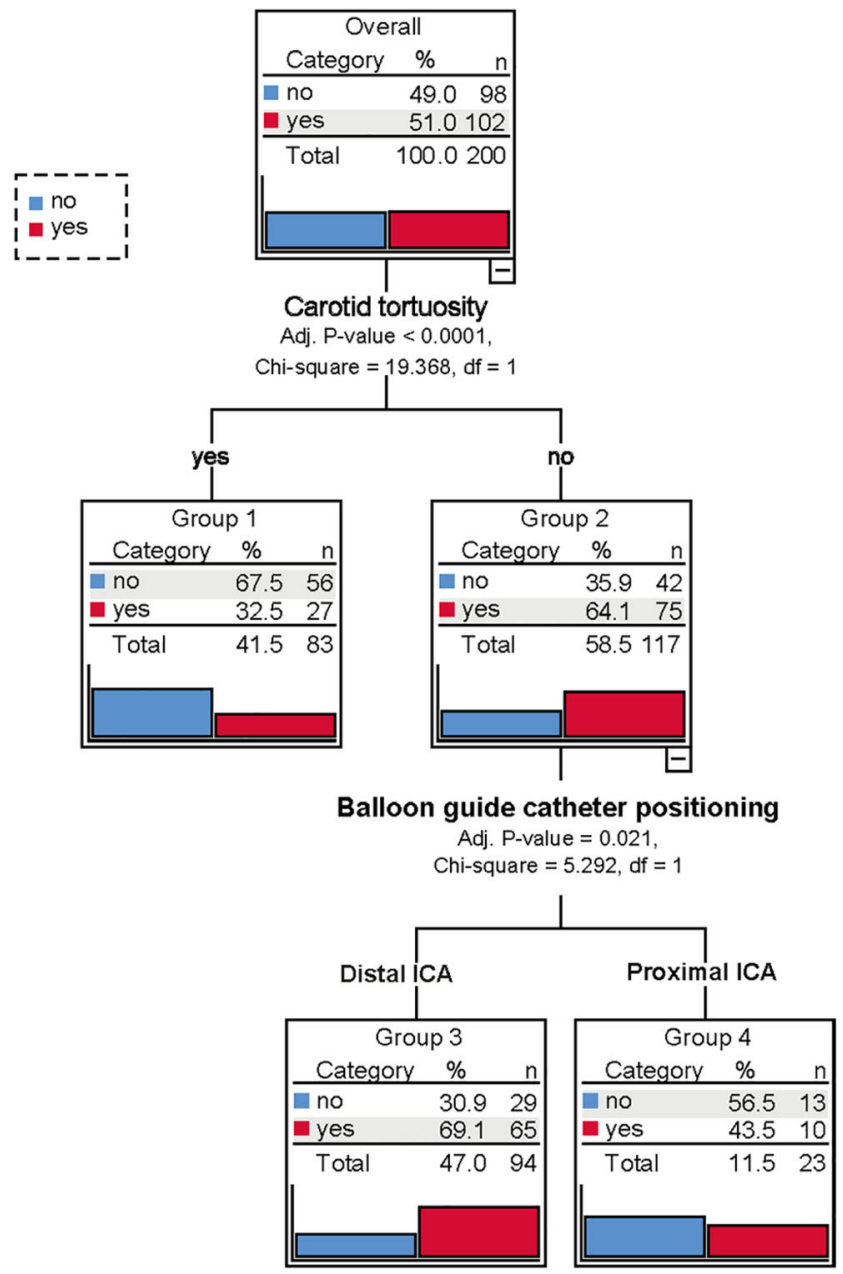

Fig. 4 Decision tree for predicting complete one-pass recanalization

the likelihood of complete first-pass recanalization with proximal BGC positioning was $43 \%$ compared with $69 \%$ for distal BGC positioning. Finally, the presence of communicating arteries that could hypothetically reduce the suction capacity of the BGC did not lower the first-pass mTICI 3 rate.

In agreement with previous studies, complete recanalization (mTICI 3, 62.5\%) was commonly achieved in our series [18]. Nevertheless, the reported rates of complete recanalization using BGC have ranged from 15 to $63 \%[8,19,20]$. We believe that these differences can be accounted for in part by differences in BGC use in the patients in these studies. That is, comparisons between BGC and other techniques are challenging when the use of flow arrest and concomitant aspiration during clot retrieval via the BGC cannot be guaranteed in all patients [7]. For example, in the ASTER trial (first-line aspiration versus first-line $\mathrm{BGC}+\mathrm{SR}$ ), the slight differences between the two treatment groups could perhaps be explained by the use of flow arrest alone without aspiration for SR thrombectomy, since there was no mention of concomitant aspiration for the BGC group in the published results [21] or in the study protocol [22].

In our study, using flow arrest and manual aspiration for retrieval in all cases, nearly $80 \%$ of all mTICI 3 outcomes were achieved with a single pass, with minimal gains in mTICI 3 outcomes after a second retrieval maneuver (13\%). This highlights the extreme importance of removing a clot completely on the very first attempt. Consequently, identifying anatomical determining factors restricting the effectiveness of MT represents an initial step towards adapting the endovascular strategy and techniques to the anatomical conditions of patients for more efficient clot extraction. While one study did report target location in the distal ICA [8], specific BGC positioning for mechanical thrombectomy has seldom been described [5, 7, 18-20]. Our study revealed an efficacy gradient depending on the location of the BGC in the carotid artery, consistent with the results of Jeong et al. [13] (TICI 3 irrespective of passes in $67 \%$ of the distal BGC group versus $45 \%$ for the proximal BGC group). Nevertheless, in their analysis of 102 thrombectomies, Jeong et al. [13] merely described the relationship between artery elongation and catheter positioning as well as the relationship between elongation and recanalization, unsupported by any statistical test results. Our study, by contrast, additionally serves to elucidate the potential success of clot removal through a BGC in diverse circumstances. This would provide us with a basis for deciding, right from the onset, when to implement the BGC technique with a distal access catheter in order to enhance the chances of achieving first-pass mTICI 3 chances in specific anatomical conditions, without having to invest time fruitlessly in the conventional "three passes and change protocol". From a femoral approach, it could be advantageous in difficult anatomical conditions to use a combination of proximal flow arrest and aspiration together with distal aspiration $+\mathrm{SR}$. This combination of techniques derives benefit from all the positive aspects of each separate method used alone [23-26]. Moreover, we commonly add a long sheath over the BGC to get more support in cases with concomitant aortoiliac elongation.

Though simultaneous use of a BGC does seen to offer enhancement [23], first-choice direct aspiration through a distal access catheter has not yet demonstrated better rates of onepass mTICI 3 than MT with SR through a BGC (concomitant flow arrest and aspiration) $[18,24]$. Among other benefits, selecting the best patient-based clot removal approach from the very beginning leads to faster interventions. This could be one of the reasons why, in their meta-analysis, Texakalidis et al. [27] disclosed increased risk of symptomatic intracerebral hemorrhage when a combined approach (distal aspiration + SR) was used as the rescue therapy after failure of primary direct aspiration. Furthermore, such other options as removing the SR delivery microcatheter prior to retrieval with aspiration have, in vitro, exhibited an absolute gain of $0.3 \mathrm{~mL} / \mathrm{s}$ in the water aspiration flow for an 8-French guide catheter and of nearly 
$1.5 \mathrm{~mL} / \mathrm{s}$ for a 5 -French distal access catheter [28]. We believe that this option is most probably unnecessary to improve aspiration capacity when larger-bore distal access catheters are used.

Something our experience has taught us is that, when performed without a distal access catheter, the removal of the SR delivery catheter before retrieval may lead to complications. Severe vasospasms or detachment of the retriever could occur because of an augmented in vivo resistance to retrieval when there is no support for the SR as recommended by the retriever manufacturers.

Finally, it has been suggested that reversed flow through the communicating arteries could reduce the suction effect, resulting in less effective clot extraction [7]. In our series, recanalization rates were not influenced by anatomical variations in the CoW. Migration into a new territory was infrequently observed (3\%); however, when adjusted for intracranial anatomy, the presence of an ACoA was found to be a protective factor against migration. We believe that aspiration through the BGC under flow arrest could indeed be beneficial by reversing the flow in the communicating arteries. What matters is not the presence of an ACoA but the hemodynamic changes that can occur through the ACoA. Thanks to the pressure gradient influenced by aspiration under flow arrest, the blood from the contralateral carotid artery can readily flow through the ACoA to the treatment side, reversing the flow in the ipsilateral A1 segment. This inverted flow, now in the direction of the ICA, could therefore prevent the clot from entering the $\mathrm{A} 1-\mathrm{A} 2$ segments during retrieval from the MCA to the ICA [26, 29-31].

\section{Limitations}

This study is a retrospective study from a single center. A significant limitation relates to the clinical outcomes, which were based on the NIHSS scores without consideration of long-term functional outcome, thus constraining the clinical value of the analysis. Furthermore, we did not collect data on the frequency of carotid dissections in relation to the position of the BGC for therapy, elongation, or the number of passes performed, and it could indeed be of interest to analyze that information together with the clinical outcomes. However, the objective of this study was focused on the factors influencing one-pass complete recanalization, currently the best angiographic outcome achievable by the fastest possible interventions. Recognizing the limitations of the technique in difficult anatomical constellations is a first step towards developing new techniques or combinations of techniques designed to enhance successful clot extraction on the first attempt.

\section{Conclusions}

In terms of clinical practice, it can be first be concluded that the chances of achieving mTICI 3 decrease substantially after the first pass, and therefore, all efforts should be aimed at attaining successful clot removal on the first attempt. Secondly, carotid tortuosity is a biomarker for an unsuccessful first pass, and in such cases, modifying the method (for example, by using a distal access catheter + flow arrest + flow reversal) should be considered in an endeavor to enhance the likelihood of first-pass mTICI 3. By means of the decision tree, when the carotid artery is elongated, the BGC technique should always be optimized, irrespective of whether or not distal BGC positioning in the ICA is feasible. Thirdly, when the carotid artery is straight, the BGC should preferably be placed in the distal (subpetrosal) ICA, because the likelihood of mTICI 3 is highest under these conditions (no tortuosity and distal $\mathrm{BGC}$ ).

Acknowledgments Open Access funding provided by Projekt DEAL Aglaé Velasco González (Neuroradiology) performed this study during a year fellowship at the Institute of Biostatistics and Clinical Research of the Faculty of Medicine, Westfälische Wilhelms-Universität Münster (WWU). The authors thank the University of Muenster (WWU) for giving us the time to complete this project. Aglaé Velasco González performed this study in the context of a program for research backed by the WWU.

\section{Compliance with Ethical Standards}

Conflict of Interest The authors declare that they have no conflicts of interest.

Open Access This article is licensed under a Creative Commons Attribution 4.0 International License, which permits use, sharing, adaptation, distribution and reproduction in any medium or format, as long as you give appropriate credit to the original author(s) and the source, provide a link to the Creative Commons licence, and indicate if changes were made. The images or other third party material in this article are included in the article's Creative Commons licence, unless indicated otherwise in a credit line to the material. If material is not included in the article's Creative Commons licence and your intended use is not permitted by statutory regulation or exceeds the permitted use, you will need to obtain permission directly from the copyright holder. To view a copy of this licence, visit http://creativecommons.org/licenses/by/4.0/.

\section{References}

1. Berkhemer OA, Fransen PS, Beumer D, van den Berg LA, Lingsma $\mathrm{HF}$, Yoo AJ, et al. A randomized trial of intraarterial treatment for acute ischemic stroke. N Engl J Med. 2015;372(1):11-20.

2. Campbell BC, Mitchell PJ, Kleinig TJ, Dewey HM, Churilov L, Yassi N, et al. Endovascular therapy for ischemic stroke with perfusion-imaging selection. N Engl J Med. 2015;372(11):1009-18.

3. Goyal M, Demchuk AM, Menon BK, Eesa M, Rempel JL, Thornton $\mathrm{J}$, et al. Randomized assessment of rapid endovascular treatment of ischemic stroke. N Engl J Med. 2015;372(11):1019-30.

4. Jovin TG, Chamorro A, Cobo E, de Miquel MA, Molina CA, Rovira A, et al. Thrombectomy within 8 hours after symptom onset in ischemic stroke. N Engl J Med. 2015;372(24):2296-306. 
5. Zaidat OO, Castonguay AC, Linfante I, Gupta R, Martin CO, Holloway WE, et al. First pass effect: a new measure for stroke thrombectomy devices. Stroke. 2018;49(3):660-6.

6. Velasco Gonzalez A, Buerke B, Gorlich D, Chapot R, Smagge L, Velasco MV, et al. Variability in the decision-making process of acute ischemic stroke in difficult clinical and radiological constellations: analysis based on a cross-sectional interview-administered stroke questionnaire. Eur Radiol. 2019;29(11):6275-84.

7. Nguyen TN, Malisch T, Castonguay AC, Gupta R, Sun CH, Martin $\mathrm{CO}$, et al. Balloon guide catheter improves revascularization and clinical outcomes with the Solitaire device: analysis of the North American Solitaire Acute Stroke Registry. Stroke. 2014;45(1):141-5.

8. Velasco A, Buerke B, Stracke CP, Berkemeyer S, Mosimann PJ, Schwindt W, et al. Comparison of a balloon guide catheter and a non-balloon guide catheter for mechanical thrombectomy. Radiology. 2016;280(1):169-76.

9. Haussen DC, Bouslama M, Grossberg JA, Nogueira RG. Remote aspiration thrombectomy in large vessel acute ischemic stroke. J Neurointerv Surg. 2017;9(3):250-2.

10. Baek JH, Kim BM, Heo JH, Nam HS, Kim YD, Park H, et al. Number of stent retriever passes associated with futile recanalization in acute stroke. Stroke. 2018;49(9):2088-95.

11. Wang D, Shu H, Meng Y, Zhang H, Wang H, He S. Factors promoting futile recanalization after stent retriever thrombectomy for stroke affecting the anterior circulation: a retrospective analysis. World Neurosurg. 2019. https://doi.org/10.1016/j.wneu.2019.09.098.

12. Yilmaz U, Muhl-Benninghaus R, Simgen A, Reith W, Korner H. Carotid elongation does not affect angiographic results of mechanical thrombectomy in acute stroke. Clin Neuroradiol. 2016;26(2):183-7.

13. Jeong DE, Kim JW, Kim BM, Hwang W, Kim DJ. Impact of balloon-guiding catheter location on recanalization in patients with acute stroke treated by mechanical thrombectomy. AJNR Am J Neuroradiol. 2019. https://doi.org/10.3174/ajnr.A6031.

14. Eckard DA, Purdy PD, Bonte FJ. Temporary balloon occlusion of the carotid artery combined with brain blood flow imaging as a test to predict tolerance prior to permanent carotid sacrifice. AJNR Am J Neuroradiol. 1992;13(6):1565-9.

15. Muller M, Hermes M, Bruckmann H, Schimrigk K. Transcranial Doppler ultrasound in the evaluation of collateral blood flow in patients with internal carotid artery occlusion: correlation with cerebral angiography. AJNR Am J Neuroradiol. 1995;16(1):195-202.

16. Tanaka H, Fujita N, Enoki T, Matsumoto K, Watanabe Y, Murase $\mathrm{K}$, et al. Relationship between variations in the circle of Willis and flow rates in internal carotid and basilar arteries determined by means of magnetic resonance imaging with semiautomated lumen segmentation: reference data from 125 healthy volunteers. AJNR Am J Neuroradiol. 2006;27(8):1770-5.

17. Berger C, Fiorelli M, Steiner T, Schabitz WR, Bozzao L, Bluhmki E, et al. Hemorrhagic transformation of ischemic brain tissue: asymptomatic or symptomatic? Stroke. 2001;32(6):1330-5.

18. Brinjikji W, Starke RM, Murad MH, Fiorella D, Pereira VM, Goyal $\mathrm{M}$, et al. Impact of balloon guide catheter on technical and clinical outcomes: a systematic review and meta-analysis. J Neurointerv Surg. 2018;10(4):335-9.

19. Zaidat OO, Mueller-Kronast NH, Hassan AE, Haussen DC, Jadhav AP, Froehler MT, et al. Impact of balloon guide catheter use on clinical and angiographic outcomes in the STRATIS Stroke Thrombectomy Registry. Stroke. 2019;50(3):697-704.
20. Nguyen TN, Castonguay AC, Nogueira RG, Haussen DC, English JD, Satti SR, et al. Effect of balloon guide catheter on clinical outcomes and reperfusion in Trevo thrombectomy. J Neurointerv Surg. 2019;11(9):861-5.

21. Lapergue B, Blanc R, Gory B, Labreuche J, Duhamel A, Marnat G, et al. Effect of endovascular contact aspiration vs stent retriever on revascularization in patients with acute ischemic stroke and large vessel occlusion: the ASTER randomized clinical trial. JAMA. 2017;318(5):443-52.

22. Lapergue B, Labreuche J, Blanc R, Barreau X, Berge J, Consoli A, et al. First-line use of contact aspiration for thrombectomy versus a stent retriever for recanalization in acute cerebral infarction: the randomized ASTER study protocol. Int J Stroke. 2018;13(1):87-95.

23. Kang DH, Kim BM, Heo JH, Nam HS, Kim YD, Hwang YH, et al. Effect of balloon guide catheter utilization on contact aspiration thrombectomy. J Neurosurg. 2018:1-7. https://doi.org/10.3171/ 2018.6.JNS181045.

24. Baek JH, Kim BM, Kang DH, Heo JH, Nam HS, Kim YD, et al. Balloon guide catheter is beneficial in endovascular treatment regardless of mechanical recanalization modality. Stroke. 2019;50(6): 1490-6.

25. Tomasello A, Ribo M, Gramegna LL, Melendez F, Rosati S, Moreu $\mathrm{M}$, et al. Procedural approaches and angiographic signs predicting first-pass recanalization in patients treated with mechanical thrombectomy for acute ischaemic stroke. Interv Neuroradiol. 2019;25(5):491-6.

26. Nikoubashman O, Wischer D, Hennemann HM, Sandmann J, Sichtermann T, Muschenich FS, et al. Balloon-guide catheters are needed for effective flow reversal during mechanical thrombectomy. AJNR Am J Neuroradiol. 2018;39(11):2011-81.

27. Texakalidis P, Giannopoulos S, Karasavvidis T, Rangel-Castilla L, Rivet DJ, Reavey-Cantwell J. Mechanical thrombectomy in acute ischemic stroke: a meta-analysis of stent retrievers vs direct aspiration vs a combined approach. Neurosurgery. 2019. https://doi.org/ $10.1093 /$ neuros/nyz258.

28. Nikoubashman O, Alt JP, Nikoubashman A, Busen M, Heringer S, Brockmann $\mathrm{C}$, et al. Optimizing endovascular stroke treatment: removing the microcatheter before clot retrieval with stentretrievers increases aspiration flow. J Neurointerv Surg. 2017;9(5):459-62.

29. Ohki T, Parodi J, Veith FJ, Bates M, Bade M, Chang D, et al. Efficacy of a proximal occlusion catheter with reversal of flow in the prevention of embolic events during carotid artery stenting: an experimental analysis. J Vasc Surg. 2001;33(3):504-9.

30. Reivich M, Holling HE, Roberts B, Toole JF. Reversal of blood flow through the vertebral artery and its effect on cerebral circulation. N Engl J Med. 1961;265:878-85.

31. Corzo H, Neidlin M, Büsen M, Nikoubashman O, Müller M, Steinseifer U, et al. 3-D computational fluid dynamics during mechanical aspiration thrombectomy in acute ischemic stroke. Neuroradiology: Springer. 2016:S1-S75. https://doi.org/10.1007/ s00234-016-1734-6.

Publisher's Note Springer Nature remains neutral with regard to jurisdictional claims in published maps and institutional affiliations. 